\title{
Hydrogen-Bridged Trinuclear Nitrosyl Carbonyl Tungstate: $\mathrm{H}_{2} \mathrm{~W}_{3}(\mathrm{CO})_{13}(\mathrm{NO})^{-}$
}

\author{
Jiann T. Lin, ", Shiow Y. Wang,"\# An C. Yeh, ${ }^{8}$ Thamas Y. R. Tsai, \$ Shie-Ming Peng, \\ Gene H. Lee, and Yuh S. Wen ${ }^{\dagger}$
}

Institute of Chemistry, Academia Sinica, Nankang, Taipei, Taiwan, Republic of China, Private Chung Chou Junior College of Technology and Commerce, Chung Hua, Taiwan, Republic of China, Department of Chemistry, Chun-Yuan Christian University, Chung-Li, Taiwan, Republic of China, and Department of Chemistry, National Taiwan University, Taipei, Republic of China

Received December 8, $1993^{\circ}$

Treatment of $\mathrm{HW}_{2}(\mathrm{CO})_{7}(\mathrm{THF})_{2}(\mathrm{NO})$ with $\mathrm{P}^{t} \mathrm{Bu}_{3}$ gives $\mathrm{HW}_{2}(\mathrm{CO})_{8}\left(\mathrm{P}^{t} \mathrm{Bu}_{3}\right)(\mathrm{NO})(1),\left[\mathrm{HP}^{t} \mathrm{PBu}_{3}\right]\left[\mathrm{HW}_{2}(\mathrm{CO})_{10}\right]$ (2), and [HPtPBu $\left.u_{3}\right]\left[(\mu-\mathrm{H})_{2} \mathrm{~W}_{3}(\mathrm{CO})_{13}(\mathrm{NO})\right](3)$. The anion $(\mu-\mathrm{H})_{2} \mathrm{~W}_{3}(\mathrm{CO})_{13}(\mathrm{NO})^{-}\left(3^{-}\right)$could be more conveniently prepared and isolated as [PPN] $\left[(\mu-\mathrm{H})_{2} \mathrm{~W}_{3}(\mathrm{CO})_{13}(\mathrm{NO})\right](4)$ from the reaction of $\mathrm{HW}_{2}(\mathrm{CO})_{9}(\mathrm{NO})$ with [PPN]$\left[\mathrm{HW}(\mathrm{CO})_{5}\right]\left(\mathrm{PPN}^{+}=\left(\mathrm{Ph}_{3} \mathrm{P}\right)_{2} \mathrm{~N}^{+}\right)$. Complex 3 reacts with a nucleophile $\mathrm{L}$ to form $\left[\mathrm{HP}^{t} \mathrm{Bu}_{3}\right]\left[(\mu-\mathrm{H})_{2} \mathrm{~W}_{3}(\mathrm{CO})_{12^{-}}\right.$ (L)(NO)] (5, L = $\left.\mathrm{PMe}_{3} ; 6, \mathrm{~L}=\mathrm{PPh}_{3} ; 7, \mathrm{~L}=\mathrm{P}(\mathrm{OEt})_{3} ; 8, \mathrm{~L}=\mathrm{MeNC}\right)$. Crystal data for 5: $\mathrm{C}_{27} \mathrm{H}_{37} \mathrm{NO}_{13} \mathrm{P}_{2} \mathrm{~W}_{3}$; $C c$ (monoclinic); $a=19.275(5), b=31.730(4), c=12.948(2) \AA ; \beta=91.22(2)^{\circ} ; Z=8 ; R=0.030, R_{\mathrm{w}}=0.030$.

\section{Introduction}

Polynuclear hydrido transition-metal complexes were subjects in several reviews. ${ }^{1}$ A three-center, two-electron (3c-2e) metalhydrogen-metal bond is frequently observed in metal to metal hydrogen transfer in polymetallic hydrides, ${ }^{2}$ and complexes containing $\mathrm{M}-\mathrm{H}-\mathrm{M}$ bonds constitute an important class among transition-metal hydrides. ${ }^{3}$ Our previous observations that $\mathrm{HW}_{2-}$ $(\mathrm{CO})_{7}\left(\mathrm{PPh}_{3}\right)_{2}(\mathrm{NO})$ readily lost one phosphine ligand in $\mathrm{THF}^{4}$ and that the substitution of $\mathrm{PCy}_{3}$ for $\mathrm{CO}$ ligands in $\mathrm{HW}_{2}(\mathrm{CO})_{9-}$ (NO) did not proceed beyond $\mathrm{HW}_{2}(\mathrm{CO})_{8}\left(\mathrm{PCy}_{3}\right)(\mathrm{NO})^{5}$ encouraged us to seek the possibility of synthesizing a coordinatively unsaturated complex, $\mathrm{HW}_{2}(\mathrm{CO})_{7}\left(\mathrm{P}^{\mathrm{B}} \mathrm{Bu} \mathrm{u}_{3}\right)(\mathrm{NO})$, by use of a sterically bulkier phosphine, $\mathrm{PtBu}_{3}$. Instead of $\mathrm{HW}_{2}(\mathrm{CO})_{7}\left(\mathrm{Pt}_{-}\right.$ $\left.\mathrm{Bu}_{3}\right)(\mathrm{NO})$, a complex $\left[\mathrm{HP}^{\mathrm{t}} \mathrm{Bu}_{3}\right]\left[(\mu-\mathrm{H})_{2} \mathrm{~W}_{3}(\mathrm{CO})_{13}(\mathrm{NO})\right]$, was isolated from the reaction of $\mathrm{HW}_{2}(\mathrm{CO})_{7}(\mathrm{THF})_{2}(\mathrm{NO})$ with $\mathrm{Pt}$. $\mathrm{Bu}_{3}$.

Polynuclear metal complexes with solely $\mathrm{M}-\mathrm{H}-\mathrm{M}$ linkages among metal atoms are very scarce. ${ }^{6}$ Although an unsupported $\mathrm{M}-\mathrm{H}-\mathrm{M}$ linkage is fairly common for dimeric group VI metal carbonyls, ${ }^{7}$ to our knowledge, $(\mu-\mathrm{H})_{2} \mathrm{~W}_{3}(\mathrm{CO})_{13}(\mathrm{NO})^{-}$is the first tungsten trimer which contains only unsupported $\mathrm{M}-\mathrm{H}-\mathrm{M}$ linkages. In this paper we describe the synthesis and the reactivity of this unusual tungsten trimer.

\section{Experimental Section}

General Procedures. All reactions and manipulations were carried out under $\mathrm{N}_{2}$ with use of standard inert-atmosphere and Schlenk techniques. Solvents were dried by standard procedures. All column chromatography was performed with use of silica gel $(230-400$ mesh

Academia Sinica.

Private Chung Chou Junior College of Science and Technology

Chun-Yuan Christian University.

II National Taiwain University.

- Abstract published in Advance ACS Abstracts, April 1, 1994.

(1) (a) Dahl, L. F. In Catalytic transition metal hydrides; Slocum, D. W., Moster, W. R., Eds.; New York Academy of Science: New York, 1983 pp 1-26. (b) Moore, D. S.; Robinson, S. D. Chem. Soc. Rev. 1983, 12 415. (c) Kaesz, H. D. J. Organomet. Chem. 1980, 200, 145.

(2) Branchadell, V. Dedieu, A. New J. Chem. 1988, 12, 443.

(3) Venanzi, L. M. Coord. Chem. Rev. 1982, 43, 251.

(4) Lin, J. T.; Huang, P. S.; Tsai, T. Y. R.; Liao, C. Y.; Tseng, L. H.; Wen, Y. S.; Shi, F. K. Inorg. Chem. 1992, $31,4444$.

(5) Lin, J, T.; Wang, S. Y.; Huang, P. S.; Hsiao, Y. M.; Wen, Y. S.; Yeh, S. K. J. Organamet. Chem. 1990, 388, 151 .

(6) (a) Masciocchi, N.; Sironi, A.; D'Afonso, G. J. Am. Chem. Soc. 1990 II2,9395. (b) Kirtley, S. W.; Olsen, J. P.; Bau, R. J. Am. Chem. Soc. $1973,95,4532$.

(7) Lin, J. T.; Yeh, A. C.; Tsai, T. Y. R.; Wen, Y. S. J. Organomet. Chem 1993, 453, 221 and references therein.
ASTM, Merck) as the stationary phase in a column $35 \mathrm{~cm}$ in length and $2.5 \mathrm{~cm}$ in diameter. $\mathrm{HW}_{2}(\mathrm{CO})_{9}(\mathrm{NO}),{ }^{8} \mathrm{HW}_{2}(\mathrm{CO})_{7}(\mathrm{THF})_{2}(\mathrm{NO}),{ }^{4}$ and $[\mathrm{PPN}]\left[\mathrm{HW}(\mathrm{CO})_{5}\right]^{9}$ were prepared by published procedures. $\mathrm{HW}_{2}(\mathrm{CO})_{9}$ ( ${ }^{15} \mathrm{NO}$ ) was prepared in the same way as $\mathrm{HW}_{2}(\mathrm{CO})_{9}(\mathrm{NO})$, except that $\mathrm{Na}^{15} \mathrm{NO}_{2}$ (99\% enriched, ICON) instead of $\mathrm{NaNO}_{2}$ was used. Infrared spectra were recorded on a Perkin-Elmer 880 spectrometer. The NMR spectra were obtained by using Bruker AMX500 ( $\left.{ }^{15} \mathrm{~N},{ }^{1} \mathrm{H},{ }^{31} \mathrm{P}\right), \mathrm{AC} 200$ $\left({ }^{1} \mathrm{H}\right)$, and $\mathrm{AC} 300\left({ }^{1} \mathrm{H},{ }^{31} \mathrm{P}\right)$ spectrometers. Elementary analyses were performed on a Perkin-Elmer $2400 \mathrm{CHN}$ analyzer.

Reaction of $\mathrm{HW}_{2}(\mathrm{CO})_{7}(\mathrm{THF})_{2}(\mathrm{NO})$ with $\mathrm{P}^{+} \mathrm{Bu}_{3}$. To a solution of $\mathrm{HW}_{2}(\mathrm{CO})_{7}(\mathrm{THF})_{2}$ (NO) $(2.6 \mathrm{~g}, 3.52 \mathrm{mmol})$ in $80 \mathrm{~mL}$ of $\mathrm{CH}_{2} \mathrm{Cl}_{2}$ was added $2.3 \mathrm{~mL}$ of THF containing $3.52 \mathrm{mmol}$ of $\mathrm{P}^{t} \mathrm{Bu}_{3}$. After the mixture was stirred for $6 \mathrm{~h}$ at room temperature, the solvent was removed in vacuo. The residue was chromatographed on a silica gel column under nitrogen. Elution with hexane gave an orange-yellow band. Further elution with $\mathrm{CH}_{2} \mathrm{Cl}_{2}$ / hexane (1:1) gave the yellow second and the orangeyellow third bands.

$\mathrm{HW}_{2}(\mathrm{CO})_{8}\left(\mathrm{P}^{t} \mathrm{Bu}_{3}\right)(\mathrm{NO})(1)$ was isolated from the first band in $25 \%$ yield. IR $\left(\mathrm{CH}_{2} \mathrm{Cl}_{2}, \mathrm{~cm}^{-1}\right): 2085$ (w), 2065 (w), 2001 (s), 1931 (vs), $\nu(\mathrm{CO}) ; 1695(\mathrm{~m}), \nu(\mathrm{NO})$. Anal. Calcd for $\mathrm{C}_{20} \mathrm{H}_{28} \mathrm{NO}_{9} \mathrm{PW}_{2}: \mathrm{C}, 29.11$; $\mathrm{H}, 3.42 ; \mathrm{N}, 1.70$. Found: $\mathrm{C}, 29.07 ; \mathrm{H}, 3.17 ; \mathrm{N}, 1.66$.

$\left[\mathrm{HP}^{1} \mathrm{Bu}_{3}\right]\left[(\mu-\mathrm{H}) \mathrm{W}_{2}(\mathrm{CO})_{10}\right](2)$ was isolated from the second band in $10 \%$ yield. The infrared $\mathrm{CO}$ stretching and ${ }^{1} \mathrm{H}$ NMR of $\mu-\mathrm{H}$ for 2 are identical to those of $\left[\mathrm{Et}_{4} \mathrm{~N}\right]\left[(\mu-\mathrm{H}) \mathrm{W}_{2}(\mathrm{CO})_{10}\right]$.

$\left[\mathrm{HP}^{t} \mathrm{Bu}_{3}\right]\left[(\mu-\mathrm{H})_{2} \mathrm{~W}_{3}(\mathrm{CO})_{13}(\mathrm{NO})\right]$ (3) was obtained from the third bands in $18.5 \%$ yield. IR $\left(\mathrm{CH}_{2} \mathrm{Cl}_{2}, \mathrm{~cm}^{-1}\right): 2080(\mathrm{w}), 2060(\mathrm{~m}), 2041$ (w), 2000 (vs), 1965 (s), 1929 (vs), 1893 (s), v(CO); 1652 (w), $(\mathrm{NO}$ ). Anal. Calcd for $\mathrm{C}_{25} \mathrm{H}_{30} \mathrm{NO}_{14} \mathrm{PW}_{3}$ : C, 26.09; H, 2.63; N, 1.22. Found: C, 26.53; H, 2.52; N, 1.05 .

[PPNI $\left.(\mu-\mathrm{H})_{2} \mathbf{W}_{3}(\mathrm{CO})_{13}(\mathrm{NO})\right]$ (4). THF $(20 \mathrm{~mL})$ was added to a flask containing $\mathrm{HW}_{2}(\mathrm{CO})_{9}(\mathrm{NO})(226 \mathrm{mg}, 34.7 \mathrm{mmol})$ and $[\mathrm{PPN}][\mathrm{HW}$. $\left.(\mathrm{CO})_{5}\right](300 \mathrm{mg}, 34.7 \mathrm{mmol})$, and the mixture was stirred at room temperature for $1.5 \mathrm{~h}$. After evaporation of the solvent, the residue was washed with hexane. The crude reaction product was then chromatographed. The orange-yellow band eluted with $\mathrm{CH}_{2} \mathrm{Cl}_{2}$ /hexane $(1: 1$ by volume) provided $310 \mathrm{mg}$ ( $72 \%$ yield) of powdery 4 . The IR spectra of 4 in $\mathrm{CO}$ and $\mathrm{NO}$ stretching region are identical to those of 3. Anal. Calcd for $\mathrm{C}_{49} \mathrm{H}_{32} \mathrm{~N}_{2} \mathrm{O}_{14} \mathrm{P}_{2} \mathrm{~W}_{3}$ : C, 39.60; H, 2.17; N, 1.88. Found: C, 39.80; $\mathrm{H}, 2.16 ; \mathrm{N}, 1.84$

$\left[\mathrm{HP}^{+} \mathrm{Bu}_{3} \mathrm{I}(\mu-\mathrm{H})_{2} \mathrm{~W}_{3}(\mathrm{CO})_{12}\left(\mathrm{PMe}_{3}\right)(\mathrm{NO})\right](5)$. A solution of $0.50 \mathrm{~g}$ of $3(0.43 \mathrm{mmol})$ in $30 \mathrm{~mL}$ of $\mathrm{CH}_{2} \mathrm{Cl}_{2}$ was stirred with excess $\mathrm{PMe}_{3}(0.23$ $\mathrm{mL}, 2.2 \mathrm{mmol}$ ) at room temperature for $18 \mathrm{~h}$. The solvent was removed, and the residue was chromatographed. Elution with hexane gave the yellow first band in a trace amount. Further elution with $\mathrm{CH}_{2} \mathrm{Cl}_{2} /$ hexane $(1: 1.5$ to $1.5: 1)$ resulted in the yellow second band and the orange-yellow

(8) Olson, J. P.; Koetzle, T. F.; Kirtley, S. W.; Andrew, M.; Tipton, D. L.; Bau, R. J. Am. Chem. Soc. 1974, 96, 6621.

(9) Darensbourg, M. Y.; Slater, S. J. Am. Chem. Soc. 1981, I03, 5914

(10) Hayter, R. G. J. Am. Chem. Soc. 1966, 88, 4376. 
Table 1. Crystallographic Data for Compound 5

\begin{tabular}{llll}
\hline chem formula & $\mathrm{C}_{27} \mathrm{H}_{37} \mathrm{NO}_{13} \mathrm{P}_{2} \mathrm{~W}_{3}$ & $\mathrm{fw}$ & 1197.07 \\
$a, \AA$ & $19.275(5)$ & space group & $C c(\mathrm{No} .9)$ \\
$b, \AA$ & $31.730(4)$ & $T,{ }^{\circ} \mathrm{C}$ & 25 \\
$c, \AA$ & $12.948(2)$ & $\lambda, \AA$ & 0.7093 \\
$\beta$, deg & $91.22(2)$ & $\rho_{\text {calcd, }} \mathrm{g} \mathrm{cm}^{-3}$ & 1.469 \\
$V, \AA^{3}$ & $7917(3)$ & $\mu, \mathrm{cm}^{-1}$ & 60.4 \\
$Z$ & 8 & transm coeff & $0.63-1.00$ \\
$R^{a}$ & 0.030 & $R_{\mathrm{w}}{ }^{b}$ & 0.030 \\
$a$ & $R=\sum|| F_{\mathrm{o}}|-| F_{\mathrm{c}}\left|/ \sum\right| F_{\mathrm{o}} \mid{ }^{b}{ }^{b} R_{\mathrm{w}}=\left[\sum w\left(F_{0}-F_{\mathrm{c}}\right)^{2} / \sum w\left|F_{\mathrm{o}}\right|^{2}\right]^{1 / 2} ; w=$ \\
$1 /\left[\sigma^{2}\left(F_{0}\right)+0.00005 F_{0}^{2}\right]$.
\end{tabular}

third band. A $14 \%$ yield ( $70 \mathrm{mg}$ ) of 3 was recovered from the second fraction. Powdery 5 was obtained from the third band in $41 \%$ yield. IR $\left(\mathrm{CH}_{2} \mathrm{Cl}_{2}, \mathrm{~cm}^{-1}\right): 2068(\mathrm{w}), 2058(\mathrm{w}), 1998(\mathrm{~ms}), 1938(\mathrm{~s}), 1909$ (sh), $v(\mathrm{CO}) ; 1609$ (w), $v(\mathrm{NO})$. Anal. Calcd for $\mathrm{C}_{27} \mathrm{H}_{39} \mathrm{NO}_{13} \mathrm{P}_{2} \mathrm{~W}_{3}: \mathrm{C}, 27.04$; $\mathrm{H}, 3.28 ; \mathrm{N}, 1.17$. Found: $\mathrm{C}, 26.76 ; \mathrm{H}, 2.84 ; \mathrm{N}, 1.15$.

The yellow-orange complexes [HP'Bu$]\left[(\mu-\mathrm{H})_{2} \mathrm{~W}_{3}(\mathrm{CO})_{12}\left(\mathrm{PPh}_{3}\right)(\mathrm{NO})\right]$ (6), $\left[\mathrm{HPtBu}_{3}\right]\left[(\mu-\mathrm{H})_{2} \mathrm{~W}_{3}(\mathrm{CO})_{12}\left(\mathrm{P}(\mathrm{OEt})_{3}\right)(\mathrm{NO})\right](7)$, and $\left[\mathrm{HP}^{t} \mathrm{Bu}_{3}\right][(\mu-$ $\left.\mathrm{H})_{2} \mathrm{~W}_{3}(\mathrm{CO})_{12}(\mathrm{MeNC})(\mathrm{NO})\right](8)$ were prepared by essentially the same procedure as for the synthesis of 5 .

Complex 6 had a yield of $45 \%$. IR $\left(\mathrm{CH}_{2} \mathrm{Cl}_{2}, \mathrm{~cm}^{-1}\right): 2068(\mathrm{w}), 2060$ (w), $2001(\mathrm{~ms}), 1933(\mathrm{~s}), 1920(\mathrm{sh}), 1885(\mathrm{sh}), \nu(\mathrm{CO}) ; 1608(\mathrm{w}), \nu(\mathrm{NO})$ Anal. Calcd for $\mathrm{C}_{42} \mathrm{H}_{45} \mathrm{NO}_{13} \mathrm{P}_{2} \mathrm{~W}_{3}: \mathrm{C}, 36.42 ; \mathrm{H}, 3.27 ; \mathrm{N}, 1.01$. Found: C, 36.28; H, 2.95; N, 0.96 .

Complex 7 had a yield of 55\%. IR $\left(\mathrm{CH}_{2} \mathrm{Cl}_{2}, \mathrm{~cm}^{-1}\right): 2069$ (w), 2059 (w), $2005(\mathrm{~ms}), 1943(\mathrm{~s}), 1931$ (s), $1879(\mathrm{sh}), \nu(\mathrm{CO}) ; 1614(\mathrm{w}), \nu(\mathrm{NO})$. Anal. Calcd for $\mathrm{C}_{30} \mathrm{H}_{45} \mathrm{NO}_{16} \mathrm{P}_{2} \mathrm{~W}_{3}: \mathrm{C}, 27.95 ; \mathrm{H}, 3.52 ; \mathrm{N}, 1.09$. Found: C, 27.73; H, 3.09; N, 1.05 .

Complex 8 had a yield of $30 \%$. IR $\left(\mathrm{CH}_{2} \mathrm{Cl}_{2}, \mathrm{~cm}^{-1}\right): 2203(\mathrm{w}), \nu(\mathrm{CN})$; 2067 (w), 2058 (w), 2008 (ms), 1970 (sh), 1943 (s), 1885 (sh), v(CO); 1618 (w) $\nu$ (NO). Anal. Caled for $\mathrm{C}_{26} \mathrm{H}_{33} \mathrm{~N}_{2} \mathrm{O}_{13} \mathrm{PW}_{3}: \mathrm{C}, 26.83 ; \mathrm{H}$, 2.86; N, 2.41. Found: $C, 26.61 ; \mathrm{H}, 2.63 ; \mathrm{N}, 2.24$.

All ${ }^{15} \mathrm{~N}$-enriched samples were prepared from $\mathrm{HW}_{2}(\mathrm{CO})_{9}\left({ }^{15} \mathrm{NO}\right)$ according to the procedures described above.

Crystallographic Studies. Crystals of 5 were grown by slow diffusion of hexane into a concentrated solution of complex 5 in $\mathrm{CH}_{2} \mathrm{Cl}_{2}$. Crystals were mounted in thin-walled glass capillaries. Diffraction measurements were made on an Enraf-Nonius CAD-4 diffractometer by using graphitemonochromated Mo $\mathrm{K} \alpha$ radiation $(\lambda=0.7093 \AA)$ with the $\theta-2 \theta$ scan mode. Unit cells were determined by centering 25 reflections in the suitable $2 \theta$ range. Other relevant experimental details are listed in Table 1. Absorption corrections (empirical method) according to $\psi$ scans of three reflections were applied. All data processing was carried out on a MICRO VAX 3600 computer by using the NRCC SDP program. ${ }^{11}$ The structures of 5 was solved by direct methods, which located the W atoms. The coordinates of all remaining atoms except hydrogen atoms were obtained from a series of structure factor calculations and Fourier syntheses. The structure was refined by minimizing $\sum w\left(\left|F_{\mathrm{o}}\right|-\left|F_{\mathrm{c}}\right|\right)^{2}$, where $w=1 / \sigma\left(F_{0}\right)^{2}$ was calculated from the counting statistics. The atomic scattering factors and anomalous dispersion terms $f^{\prime}$ and $f^{\prime \prime}$ were taken from ref 12. All non-hydrogen atoms were refined anisotropically. All hydrogen atoms except for $\mathrm{H}-\mathrm{P}$ were included in the structure factor calculation in idealized positions with $d_{\mathrm{C}-\mathrm{H}}=0.98 \AA$. The final positional parameters are listed in Table 2, and selected interatomic distances and bond angles are given in Table 3.

\section{Results and Discussion}

Equation 1 describes the synthesis of $(\mu-\mathrm{H})_{2} \mathrm{~W}_{3}(\mathrm{CO})_{13}(\mathrm{NO})^{-}$ (3-) in this study. Formation of $3^{-}$from $\mathrm{HW}_{2}(\mathrm{CO})_{7}(\mathrm{THF})_{2}(\mathrm{NO})$

$$
\begin{gathered}
\mathrm{HW}_{2}(\mathrm{CO})_{7}(\mathrm{THF})_{2}(\mathrm{NO})+\mathrm{P}^{\mathrm{t}} \mathrm{Bu}_{3} \stackrel{\mathrm{CH}_{2} \mathrm{Cl}_{2}}{\rightarrow} \\
{\left[\mathrm{HP}^{\mathrm{t}} \mathrm{Bu}_{3}\right]\left[(\mu-\mathrm{H})_{2} \mathrm{~W}_{3}(\mathrm{CO})_{13}(\mathrm{NO})\right]+} \\
\mathrm{HW}_{2}(\mathrm{CO})_{8}\left(\mathrm{P}^{\mathrm{t} B \mathrm{Bu}_{3}}\right)(\mathrm{NO})+\left[\mathrm{HP}^{\mathrm{t}} \mathrm{Bu}_{3}\right]\left[\mathrm{HW}_{2}(\mathrm{CO})_{10}\right]
\end{gathered}
$$

or $\mathrm{HW}_{2}(\mathrm{CO})_{9}(\mathrm{NO})$ and $\mathrm{PBu}_{3}$ is likely via electron transfer from $\mathrm{P}^{2} \mathrm{Bu}_{3}$ to the tungsten dimer followed by fragmentation of the dimer to $\mathrm{HW}(\mathrm{CO})_{5}{ }^{-}$. Although there is no direct evidence of

(11) (a) Gabe, E. J.; Lee, F. L. Acta Crystallogr. 1981, 37B, 339. (b) Gabe, E. J.; LePage, Y.; White, P. S.; Lee, F. L. Acta Crystallogr. 1987, 43C 294.

(12) International Tables for X-ray Crystallography; Kynoch: Birmingham,

\begin{tabular}{|c|c|c|c|c|}
\hline atom & $x$ & $y$ & $z$ & $B_{i 10}, \AA^{2}$ \\
\hline $\mathrm{W}(1 \mathrm{~A})$ & $0.01332(5)$ & $0.38887(3)$ & $0.30048(7)$ & $3.83(4)$ \\
\hline $\mathrm{W}(2 \mathrm{~A})$ & $0.06803(5)$ & $0.31736(3)$ & $0.49077(7)$ & $3.90(4)$ \\
\hline $\mathrm{W}(3 \mathrm{~A})$ & $0.16537(5)$ & $0.43982(3)$ & $0.24949(8)$ & $4.49(4)$ \\
\hline $\mathrm{P}(1 \mathrm{~A})$ & $-0.0330(3)$ & $0.4386(2)$ & $0.4368(5)$ & $5.4(3)$ \\
\hline $\mathrm{N}(1 \mathrm{~A})$ & $-0.0230(8)$ & $0.4218(5)$ & $0.2028(13)$ & $5.0(8)$ \\
\hline $\mathrm{C}(1 \mathrm{~A})$ & $-0.0674(12)$ & $0.3561(6)$ & $0.3081(17)$ & $5.3(11)$ \\
\hline $\mathrm{C}(2 \mathrm{~A})$ & $0.0561(11)$ & $0.3503(7)$ & 0.2022 (15) & $5.5(11)$ \\
\hline $\mathrm{C}(3 \mathrm{~A})$ & $-0.0321(11)$ & $0.3075(7)$ & $0.5221(17)$ & $5.4(12)$ \\
\hline $\mathrm{C}(4 \mathrm{~A})$ & $0.0766(11)$ & $0.3543(7)$ & $0.6231(16)$ & $5.5(11)$ \\
\hline$C(5 A)$ & $0.0932(12)$ & $0.2692(8)$ & $0.5793(16)$ & $6.0(12)$ \\
\hline $\mathrm{C}(6 \mathrm{~A})$ & $0.1715(11)$ & $0.3312(6)$ & $0.4693(16)$ & $5.1(11)$ \\
\hline$C(7 A)$ & $0.0593(10)$ & $0.2849(9)$ & $0.3756(18)$ & $7.6(16)$ \\
\hline $\mathrm{C}(8 \mathrm{~A})$ & $0.2051(15)$ & $0.4457(8)$ & $0.3936(20)$ & $8.6(17)$ \\
\hline $\mathrm{C}(9 \mathrm{~A})$ & $0.2075(10)$ & $0.3871(8)$ & $0.2390(19)$ & $6.4(13)$ \\
\hline$C(10 A)$ & $0.2498(12)$ & $0.4560(8)$ & $0.1945(18)$ & $6.6(14)$ \\
\hline C(11A) & $0.1198(11)$ & $0.4342(8)$ & $0.1095(15)$ & $5.5(11)$ \\
\hline$C(12 A)$ & $0.1200(12)$ & $0.4979(8)$ & $0.2716(16)$ & $5.8(12)$ \\
\hline$C(13 A)$ & $0.0318(16)$ & $0.4658(8)$ & $0.5200(21)$ & $8.6(17)$ \\
\hline$C(14 A)$ & $-0.0779(18)$ & $0.4821(10)$ & $0.3835(25)$ & $12.6(24)$ \\
\hline$C(15 A)$ & $-0.0866(16)$ & $0.4192(10)$ & $0.5335(25)$ & $11.2(21)$ \\
\hline$O(1 \mathrm{~A})$ & $-0.1209(9)$ & $0.3358(6)$ & $0.3095(15)$ & $9.6(12)$ \\
\hline$O(2 \mathrm{~A})$ & $0.0799(9)$ & $0.3287(5)$ & $0.1447(13)$ & $7.9(10)$ \\
\hline $\mathrm{O}(3 \mathrm{~A})$ & $-0.0881(8)$ & $0.2984(5)$ & $0.5438(12)$ & $7.3(9)$ \\
\hline$O(4 A)$ & $0.0835(9)$ & $0.3754(6)$ & $0.6967(13)$ & $8.3(10)$ \\
\hline$O(5 \mathrm{~A})$ & $0.1080(9)$ & $0.2397(6)$ & $0.6286(12)$ & $8.5(10)$ \\
\hline$O(6 \mathrm{~A})$ & $0.2276(7)$ & $0.3386(5)$ & $0.4643(14)$ & $7.7(11)$ \\
\hline$O(7 \mathrm{~A})$ & $0.0559(9)$ & $0.2568(5)$ & $0.3094(12)$ & $7.6(10)$ \\
\hline$O(8 \mathrm{~A})$ & $0.2306(10)$ & $0.4507(6)$ & $0.4776(14)$ & $10.2(12)$ \\
\hline$O(9 A)$ & $0.2347(9)$ & $0.3528(6)$ & $0.2276(13)$ & $8.9(11)$ \\
\hline $\mathrm{O}(10 \mathrm{~A})$ & $0.2980(8)$ & $0.4781(7)$ & $0.1589(15)$ & $9.9(12)$ \\
\hline O(11A) & $0.0954(9)$ & $0.4319(7)$ & $0.0308(12)$ & $9.2(12)$ \\
\hline$O(12 A)$ & $0.0958(11)$ & $0.5294(5)$ & $0.2766(14)$ & $10.2(13)$ \\
\hline$O(13 A)$ & $-0.0490(9)$ & $0.4456(5)$ & $0.1341(12)$ & $7.7(10)$ \\
\hline $\mathrm{W}(1 \mathrm{~B})$ & 0.01585 & $0.11440(3)$ & 1.04898 & $3.50(4)$ \\
\hline $\mathrm{W}(2 \mathrm{~B})$ & $0.07489(5)$ & $0.20547(3)$ & $0.93425(7)$ & $4.43(4)$ \\
\hline $\mathrm{W}(3 \mathrm{~B})$ & $0.16222(5)$ & $0.05305(3)$ & $1.07299(8)$ & $4.91(5)$ \\
\hline$P(\mathrm{IB})$ & $-0.0302(4)$ & $0.0808(2)$ & $0.8903(5)$ & $6.0(3)$ \\
\hline $\mathrm{N}(1 \mathrm{~B})$ & $-0.0648(9)$ & $0.1452(6)$ & $1.0632(14)$ & $5.8(10)$ \\
\hline C(1B) & $-0.0248(9)$ & $0.0703(6)$ & $1.1301(15)$ & $3.9(10)$ \\
\hline$C(2 B)$ & $0.0553(13)$ & $0.1414(7)$ & $1.1749(16)$ & $6.4(13)$ \\
\hline $\mathrm{C}(3 \mathrm{~B})$ & 0.0079 (11) & $0.2285(6)$ & 1.0409 (16) & $4.8(11)$ \\
\hline C (4B) & 0.0039 (12) & $0.2022(7)$ & $0.8274(16)$ & $5.8(13)$ \\
\hline$C(5 B)$ & $0.0920(10)$ & $0.2649(8)$ & $0.8925(17)$ & $6.0(13)$ \\
\hline $\mathrm{C}(6 \mathrm{~B})$ & $0.1508(13)$ & $0.1828(8)$ & (18) & $6.7(14)$ \\
\hline C(7B) & $0.1494(13)$ & $0.2151(8)$ & $1.0492(16)$ & $6.2(13)$ \\
\hline $\mathrm{C}(8 \mathrm{~B})$ & 0.1939 (12) & $0.0478(8)$ & $0.9385(23)$ & $7.7(16)$ \\
\hline $\mathrm{C}(9 \mathrm{~B})$ & $0.2187(11)$ & $0.1077(9)$ & $15(21)$ & $7.7(16)$ \\
\hline $\mathrm{C}(10 \mathrm{~B})$ & $0.2470(14)$ & $0.0209(9)$ & $1.1261(20)$ & $7.8(16)$ \\
\hline $\mathrm{C}(11 \mathrm{~B})$ & $0.1338(12)$ & $0.0542(8)$ & $1.2226(18)$ & $6.9(13)$ \\
\hline$C(12 B)$ & $0.1112(13)$ & $-0.0009(8)$ & $1.0631(19)$ & $7.4(15)$ \\
\hline $\mathrm{C}(13 \mathrm{~B})$ & $0.0228(12)$ & $0.0842(10)$ & $0.7768(18)$ & $8.2(17)$ \\
\hline$C(14 B)$ & $-0.0596(23)$ & $0.0251(9)$ & $0.9003(30)$ & $15.2(29)$ \\
\hline$C(15 B)$ & $-0.1145(15)$ & 0.1009 (11) & $0.8445(18)$ & $9.9(21)$ \\
\hline $\mathrm{O}(1 \mathrm{~B})$ & $0.0454(9)$ & $0.0419(6)$ & $1.1815(14)$ & $8.6(11)$ \\
\hline $\mathrm{O}(2 \mathrm{~B})$ & $0.0828(9)$ & $0.1575(5)$ & $1.2512(11)$ & $6.9(9)$ \\
\hline$O(3 B)$ & $-0.0311(8)$ & $0.2442(5)$ & $1.0981(11)$ & $6.5(9)$ \\
\hline$O(4 B)$ & $-0.0370(9)$ & $0.2008(6)$ & $0.7627(13)$ & $9.2(11)$ \\
\hline$O(5 B)$ & $0.1012(9)$ & $0.2997(6)$ & (13) & $8.8(11)$ \\
\hline$O(6 B)$ & $0.1905(10)$ & $0.1704(7)$ & $0.7908(15)$ & $11.1(14)$ \\
\hline O(7B) & $0.1878(10)$ & $0.2246(6)$ & $1.1159(13)$ & 9.3 (i1) \\
\hline $\mathrm{O}(8 \mathrm{~B})$ & $0.2148(13)$ & $0.0458(8)$ & $0.8517(16)$ & $13.7(17)$ \\
\hline $\mathrm{O}(9 \mathrm{~B})$ & $0.2517(11)$ & $0.1356(7)$ & $1.1146(21)$ & $13.8(18)$ \\
\hline $\mathrm{O}(10 \mathrm{~B})$ & 0.298 & $0.0061(7)$ & $1.1535(16)$ & $10.5(13)$ \\
\hline $\mathrm{O}(11 \mathrm{~B})$ & $0.1118(10)$ & $0.0535(7)$ & $1.3082(13)$ & $9.9(13)$ \\
\hline $\mathrm{O}(12 \mathrm{~B})$ & $0.0832(9)$ & $-0.0338(5)$ & $1.0574(14)$ & $8.5(12)$ \\
\hline$O(13 B)$ & $-0.1202(7)$ & $0.1629(6)$ & $1.0662(13)$ & $7.6(10)$ \\
\hline$P(1 C)$ & $0.8520(3)$ & $0.1450(2)$ & $0.3536(4)$ & $4.3(3)$ \\
\hline $\mathrm{P}(2 \mathrm{C})$ & $0.3060(3)$ & $0.1536(2)$ & $0.4 C$ & $4.9(3)$ \\
\hline C(IC) & $0.7862(11)$ & $0.1023(7)$ & (14) & $5.1(11)$ \\
\hline $\mathrm{C}(2 \mathrm{C})$ & $0.7232(14)$ & $0.1093(9)$ & $0.4052(21)$ & $8.2(16)$ \\
\hline$C(3 C)$ & $0.7675(15)$ & $0.0987(8)$ & $0.2239(20)$ & $8.1(16)$ \\
\hline $\mathrm{C}(4 \mathrm{C})$ & $0.8117(14)$ & $0.0579(8)$ & $0.3725(22)$ & $8.6(17)$ \\
\hline $\mathrm{C}(5 \mathrm{C})$ & $0.9126(12)$ & $0.1373(8)$ & $0.4631(17)$ & $6.3(13)$ \\
\hline$C(6 C)$ & $0.8737(14)$ & $0.1295(11)$ & $0.5624(16)$ & $9.4(19)$ \\
\hline $\mathrm{C}(7 \mathrm{C})$ & 0.9609 (14) & $0.0985(9$ & $0.4362(21)$ & $8.5(16)$ \\
\hline$C(8 C)$ & $0.9630(14)$ & $0.1776(10)$ & $0.4787(20)$ & $8.9(17)$ \\
\hline $\mathrm{C}(9 \mathrm{C})$ & $0.8165(10)$ & 0.1994 & $0.3409(16)$ & $5.1(11)$ \\
\hline $\mathrm{C}(10 \mathrm{C}$ & $0.7594(14)$ & & $5(21)$ & $8.9(17)$ \\
\hline C(11C) & $0.7888(17)$ & $0.2145(8)$ & $0.4469(25)$ & $10.9(20)$ \\
\hline$C(12 \mathrm{C})$ & $0.8784(17)$ & $0.2291(10)$ & $0.3103(25)$ & $11.3(21)$ \\
\hline $\mathrm{C}(13 \mathrm{C}$ & $0.2616(12)$ & $0.1154(8)$ & $0.4984(21)$ & $7.4(15)$ \\
\hline$C(14 C)$ & $0.1871(13)$ & $0.1300(10)$ & $0.5143(20)$ & $8.2(17)$ \\
\hline$C(15 C)$ & $0.2977(17)$ & $0.1108(10)$ & $0.5935(21)$ & $9.7(19)$ \\
\hline$C(16 C)$ & $0.2613(23)$ & $0.0709(8)$ & $0.4426(32)$ & $14.5(27)$ \\
\hline $\mathrm{c}(17 \mathrm{C}$ & $0.4013(12)$ & $0.1406(8)$ & $0.3883(16)$ & $6.2(13)$ \\
\hline $\mathrm{C}(18 \mathrm{C})$ & $0.4229(18)$ & $0.1596(11)$ & $0.2876(24)$ & $11.5(23)$ \\
\hline C(19C) & $0.4451(14)$ & $0.1551(10)$ & $0.4817(22)$ & $9.3(19)$ \\
\hline$C(20 C)$ & $0.4154(15)$ & $0.0910(8)$ & $0.3846(29)$ & $10.4(22)$ \\
\hline$C(21 C)$ & $0.2871(12)$ & $0.2096(7)$ & $0.4295(19)$ & $6.3(13)$ \\
\hline$C(22 \mathrm{C})$ & $0.3311(15)$ & $0.2367(9)$ & $0.3703(30)$ & $12.2(25)$ \\
\hline$C(23 C)$ & $0.2905(16)$ & $0.2188(9)$ & $0.5451(23)$ & $10.3(19)$ \\
\hline$C(24 C)$ & $0.2138(15)$ & $0.2187(10)$ & $0.3855(27)$ & $11.6(22)$ \\
\hline
\end{tabular}
England, 1974; Vol. IV.
Table 2. Positional Parameters and $\beta_{\text {iso }}$ Values for the Atoms in 5 
Table 3. Selected Bond Distances ( $\AA$ ) and Angles (deg) for Complexes 5

\begin{tabular}{|c|c|c|c|}
\hline \multicolumn{4}{|c|}{ Distances } \\
\hline$W(1 A)-W(2 A)$ & $3.492(2)$ & $W(1 B)-W(2 B)$ & $3.451(2)$ \\
\hline$W(1 A)-W(3 A)$ & $3.422(2)$ & $W(1 B)-W(3 B)$ & $3.436(2)$ \\
\hline $\mathrm{W}(1 \mathrm{~A})-\mathrm{C}(1 \mathrm{~A})$ & $1.92(3)$ & $W(1 B)-C(1 B)$ & $1.91(2)$ \\
\hline$W(1 \mathrm{~A})-C(2 \mathrm{~A})$ & $1.86(3)$ & $W(1 B)-C(2 B)$ & $1.98(2)$ \\
\hline $\mathrm{W}(1 \mathrm{~A})-\mathrm{N}(1 \mathrm{~A})$ & $1.76(3)$ & $W(1 B)-N(1 B)$ & $1.85(2)$ \\
\hline$W(1 A)-P(1 A)$ & $2.549(8)$ & $W(1 B)-P(1 B)$ & $2.483(7)$ \\
\hline$W(2 A)-C(3 A)$ & $1.97(3)$ & $W(2 B)-C(3 B)$ & $2.03(3)$ \\
\hline $\mathrm{W}(2 \mathrm{~A})-\mathrm{C}(4 \mathrm{~A})$ & $2.06(3)$ & $\mathrm{W}(2 \mathrm{~B})-\mathrm{C}(4 \mathrm{~B})$ & $1.92(3)$ \\
\hline$W(2 A)-C(5 A)$ & $1.94(3)$ & $W(2 B)-C(5 B)$ & $1.90(4)$ \\
\hline$W(2 A)-C(6 A)$ & $2.02(3)$ & $W(2 B)-C(6 B)$ & $1.98(3)$ \\
\hline$W(2 A)-C(7 A)$ & $1.82(3)$ & $W(2 B)-C(7 B)$ & $2.03(3)$ \\
\hline$W(3 A)-C(8 A)$ & $1.96(4)$ & $W(3 B)-C(8 B)$ & $1.72(4)$ \\
\hline$W(3 A)-C(9 A)$ & $1.81(3)$ & $W(3 B)-C(9 B)$ & $1.93(3)$ \\
\hline $\mathrm{W}(3 \mathrm{~A})-\mathrm{C}(10 \mathrm{~A})$ & $1.96(3)$ & $W(3 B)-C(10 B)$ & $1.96(4)$ \\
\hline $\mathrm{W}(3 \mathrm{~A})-\mathrm{C}(11 \mathrm{~A})$ & $1.98(3)$ & $W(3 B)-C(11 B)$ & $1.98(4)$ \\
\hline $\mathrm{W}(3 \mathrm{~A})-\mathrm{C}(12 \mathrm{~A})$ & $2.04(3)$ & $\mathrm{W}(3 \mathrm{~B})-\mathrm{C}(12 \mathrm{~B})$ & $1.94(4)$ \\
\hline$N(1 A)-O(13 A)$ & $1.27(3)$ & $N(1 B)-O(13 B)$ & $1.19(3)$ \\
\hline$C(1 \mathrm{~A})-O(1 \mathrm{~A})$ & $1.18(3)$ & $C(1 B)-O(1 B)$ & $1.18(3)$ \\
\hline$C(2 A)-O(2 A)$ & $1.21(4)$ & $\mathrm{C}(2 \mathrm{~B})-\mathrm{O}(2 \mathrm{~B})$ & $1.19(3)$ \\
\hline$C(3 A)-O(3 A)$ & $1.22(4)$ & $C(3 B)-O(3 B)$ & $1.19(3)$ \\
\hline$C(4 A)-O(4 A)$ & $1.15(4)$ & $C(4 B)-O(4 B)$ & $1.16(3)$ \\
\hline $\mathrm{C}(5 \mathrm{~A})-\mathrm{O}(5 \mathrm{~A})$ & $1.21(3)$ & $C(5 B)-O(5 B)$ & $1.21(5)$ \\
\hline$C(6 A)-O(6 A)$ & $1.11(3)$ & $C(6 B)-O(6 B)$ & $1.15(3)$ \\
\hline $\mathrm{C}(7 \mathrm{~A})-\mathrm{O}(7 \mathrm{~A})$ & $1.25(4)$ & $\mathrm{C}(7 \mathrm{~B})-\mathrm{O}(7 \mathrm{~B})$ & $1.17(4)$ \\
\hline$C(8 \mathrm{~A})-O(8 \mathrm{~A})$ & $1.22(4)$ & $\mathrm{C}(8 \mathrm{~B})-\mathrm{O}(8 \mathrm{~B})$ & $1.34(5)$ \\
\hline$C(9 A)-O(9 A)$ & $1.28(4)$ & $\mathrm{C}(9 \mathrm{~B})-\mathrm{O}(9 \mathrm{~B})$ & $1.18(4)$ \\
\hline$C(10 A)-O(10 A)$ & $1.16(4)$ & $C(10 \mathrm{~B})-\mathrm{O}(10 \mathrm{~B})$ & $1.18(4)$ \\
\hline$C(11 A)-O(11 A)$ & $1.19(3)$ & $C(11 B)-O(11 B)$ & $1.24(5)$ \\
\hline$C(12 A)-O(12 A)$ & $1.12(4)$ & $C(12 B)-O(12 B)$ & $1.16(5)$ \\
\hline$P(1 C)-H P(1 C)$ & 1.08 & $\mathrm{P}(2 \mathrm{C})-\mathrm{HP}(2 \mathrm{C})$ & 1.06 \\
\hline \multicolumn{4}{|c|}{ Angl } \\
\hline$W(2 A)-W(1 A)-W(3 A)$ & $101.22(4)$ & $W(2 B)-W(1 B)-W(3 B)$ & $103.68(3)$ \\
\hline$W(1 \mathrm{~A})-\mathrm{C}(1 \mathrm{~A})-\mathrm{O}(\mathrm{A})$ & $178(3)$ & $W(1 B)-C(1 B)-O(B)$ & $177(2)$ \\
\hline $\mathrm{W}(1 \mathrm{~A})-\mathrm{C}(2 \mathrm{~A})-\mathrm{O}(2 \mathrm{~A})$ & $176(2)$ & $W(1 B)-C(2 B)-O(2 B)$ & $179(2)$ \\
\hline $\mathrm{W}(1 \mathrm{~A})-\mathrm{N}(1 \mathrm{~A})-\mathrm{O}(13 \mathrm{~A})$ & $177(2)$ & $\mathrm{W}(1 \mathrm{~B})-\mathrm{N}(1 \mathrm{~B})-\mathrm{O}(13 \mathrm{~B})$ & $178(2)$ \\
\hline $\mathrm{W}(2 \mathrm{~A})-\mathrm{C}(3 \mathrm{~A})-\mathrm{O}(3 \mathrm{~A})$ & $172(3)$ & $W(2 B)-C(3 B)-O(3 B)$ & $177(2)$ \\
\hline$W(2 A)-C(4 A)-O(4 A)$ & $176(2)$ & $W(2 B)-C(4 B)-O(4 B)$ & $176(2)$ \\
\hline $\mathrm{W}(2 \mathrm{~A})-\mathrm{C}(5 \mathrm{~A})-\mathrm{O}(5 \mathrm{~A})$ & $173(2)$ & $W(2 B)-C(5 B)-O(5 B)$ & $177(2)$ \\
\hline$W(2 A)-C(6 A)-O(6 A)$ & $178(3)$ & $W(2 B)-C(6 B)-O(6 B)$ & $176(3)$ \\
\hline$W(2 A)-C(7 A)-O(7 A)$ & $175(2)$ & $W(2 B)-C(7 B)-O(7 B)$ & $174(3)$ \\
\hline$W(3 A)-C(8 A)-O\left({ }^{*} A\right)$ & $174(3)$ & $W(3 B)-C(8 B)-O(8 B)$ & $176(2)$ \\
\hline $\mathrm{W}(3 \mathrm{~A})-\mathrm{C}(9 \mathrm{~A})-\mathrm{O}(9 \mathrm{~A})$ & $176(2)$ & $W(3 B)-C(9 B)-O(9 B)$ & $175(2)$ \\
\hline $\mathrm{W}(3 \mathrm{~A})-\mathrm{C}(10 \mathrm{~A})-\mathrm{O}(11 \mathrm{~A})$ & $173(3)$ & $W(3 B)-C(10 B)-O(10 B)$ & $175(3)$ \\
\hline $\mathrm{W}(3 \mathrm{~A})-\mathrm{C}(11 \mathrm{~A})-\mathrm{O}(11 \mathrm{~A})$ & $175(2)$ & $W(3 B)-C(11 B)-O(11 B)$ & $173(2)$ \\
\hline $\mathrm{W}(3 \mathrm{~A})-\mathrm{C}(12 \mathrm{~A})-\mathrm{O}(12 \mathrm{~A})$ & $176(2)$ & $W(3 B)-C(12 B)-O(12 B)$ & $173(3)$ \\
\hline $\mathbf{P}(1 \mathrm{~A})-\mathbf{W}(1 \mathrm{~A})-\mathbf{N}(1 \mathrm{~A})$ & $90.4(8)$ & $P(1 B)-W(1 B)-N(1 B)$ & $90.6(6)$ \\
\hline$P(1 A)-W(1 A)-C(1 A)$ & $90(1)$ & $P(1 B)-W(1 B)-C(1 B)$ & $90.1(8)$ \\
\hline$P(1 A)-W(1 A)-C(2 A)$ & $175.6(9)$ & $P(1 B)-W(1 B)-C(2 B)$ & $177.0(7)$ \\
\hline$N(1 A)-W(1 A)-C(1 A)$ & $92(1)$ & $N(1 B)-W(1 B)-C(1 B)$ & $89.4(9)$ \\
\hline$N(1 A)-W(1 A)-C(2 A)$ & $93(1)$ & $\mathrm{N}(1 \mathrm{~B})-\mathrm{W}(1 \mathrm{~B})-\mathrm{C}(2 \mathrm{~B})$ & 92(1) \\
\hline$C(1 \mathrm{~A})-\mathrm{W}(1 \mathrm{~A})-\mathrm{C}(2 \mathrm{~A})$ & 92(1) & $C(1 B)-W(1 B)-C(2 B)$ & $92(1)$ \\
\hline$C(3 A)-W(2 A)-C(4 A)$ & $89(1)$ & $C(3 B)-W(2 B)-C(4 B)$ & $93(1)$ \\
\hline$C(3 A)-W(2 A)-C(5 A)$ & $88(1)$ & $C(3 B)-W(2 B)-C(5 B)$ & $88(1)$ \\
\hline$C(3 A)-W(2 A)-C(6 A)$ & $173(1)$ & $C(3 B)-W(2 B)-C(6 B)$ & $172(1)$ \\
\hline$C(3 A)-W(2 A)-C(7 A)$ & $90(1)$ & $C(3 B)-W(2 B)-C(7 B)$ & $84(1)$ \\
\hline$C(4 A)-W(2 A)-C(5 A)$ & $87(1)$ & $\mathrm{C}(4 \mathrm{~B})-\mathrm{W}(2 \mathrm{~B})-\mathrm{C}(5 \mathrm{~B})$ & $88(1)$ \\
\hline$C(4 A)-W(2 A)-C(6 A)$ & $85(1)$ & $C(4 B)-W(2 B)-C(6 B)$ & $95(1)$ \\
\hline$C(4 A)-W(2 A)-C(7 A)$ & $177(1)$ & $C(4 B)-W(2 B)-C(7 B)$ & $175(1)$ \\
\hline$C(5 A)-W(2 A)-C(6 A)$ & 92(1) & $C(5 B)-W(2 B)-C(6 B)$ & 92(1) \\
\hline$C(5 A)-W(2 A)-C(7 A)$ & $91(1)$ & $C(5 B)-W(2 B)-C(7 B)$ & $88(1)$ \\
\hline$C(6 A)-W(2 A)-C(7 A)$ & $97(1)$ & $C(6 B)-W(2 B)-C(7 B)$ & $89(1)$ \\
\hline$C(8 A)-W(3 A)-C(9 A)$ & $89(2)$ & $C(8 B)-W(3 B)-C(9 B)$ & $94(1)$ \\
\hline$C(8 A)-W(3 A)-C(10 A)$ & $91(1)$ & $C(8 B)-W(3 B)-C(10 B)$ & $91(1)$ \\
\hline$C(8 A O-W(3 A)-C(11 A)$ & $179(1)$ & $C(8 B)-W(3 B)-C(11 B)$ & $174(1)$ \\
\hline$C(8 A)-W(3 A)-C(12 A)$ & $88(1)$ & $\mathrm{C}(8 \mathrm{~B})-\mathrm{W}(3 \mathrm{~B})-\mathrm{C}(12 \mathrm{~B})$ & $91(1)$ \\
\hline$C(9 A)-W(3 A)-C(10 A)$ & $89(1)$ & $C(9 B)-W(3 B)-C(10 B)$ & $88(1)$ \\
\hline$C(9 A)-W(3 A)-C(11 A)$ & $92(1)$ & $C(9 B)-W(3 B)-C(11 B)$ & $91(1)$ \\
\hline $\mathrm{C}(9 \mathrm{~A})-\mathrm{W}(3 \mathrm{~A})-\mathrm{C}(12 \mathrm{~A})$ & $177(1)$ & $C(9 B)-W(3 B)-C(11 B)$ & $175(1)$ \\
\hline$C(10 A)-W(3 A)-C(11 A)$ & $90(1)$ & $C(10 B)-W(3 B)-C(11 B)$ & $86(1)$ \\
\hline$C(10 A)-W(3 A)-C(12 A)$ & $90(1)$ & $C(10 B)-W(3 B)-C(12 B)$ & $90(1)$ \\
\hline$C(11 A)-W(3 A)-C(12 A)$ & $92(1)$ & $C(11 B)-W(3 B)-C(12 B)$ & $84(1)$ \\
\hline
\end{tabular}

formation of $\mathrm{HW}(\mathrm{CO})_{5}$, bona fide $\mathrm{HW}(\mathrm{CO})_{5}$ indeed reacts with $\mathrm{HW}_{2}(\mathrm{CO})_{9}(\mathrm{NO})$ in THF to provide $3^{-}$in high yield (eq 2 ).

$$
\begin{aligned}
\mathrm{HW}_{2}(\mathrm{CO})_{9}(\mathrm{NO})+[\mathrm{PPN}]\left[\mathrm{HW}(\mathrm{CO})_{5}\right] \stackrel{\mathrm{THF}}{\rightarrow} \\
{[\mathrm{PPN}]\left[(\mu-\mathrm{H})_{2} \mathrm{~W}_{3}(\mathrm{CO})_{13}(\mathrm{NO})\right] }
\end{aligned}
$$

It is very common for phosphines to function as electron-transfer reagents toward organometallic complexes. ${ }^{13}$ Coordination of $\mathrm{P}^{\prime} \mathrm{Bu}_{3}$ competes effectively with electron-transfer processes; therefore, complex 1 is the predominant product if more than 1.5 equiv of $\mathrm{P}^{t} \mathrm{Bu}_{3}$ is used. Other reducing agents, such as $\mathrm{Na} / \mathrm{Ph}_{2}$ $\mathrm{CO}, \mathrm{BuLi}$, and $\mathrm{Cp}_{2} \mathrm{Co}$, also react with $\mathrm{HW}_{2}(\mathrm{CO})_{9}(\mathrm{NO})$ to form $3^{-}{ }^{14}$ Decomposition of both $\mathrm{HW}(\mathrm{CO})_{5}^{-15}$ and $3^{-}$will afford $\mathrm{HW}_{2}(\mathrm{CO})_{10^{-}}$.

Molecular Structure of $\left[\mathrm{HP}^{t} \mathrm{Bu}_{3}\right]\left[(\mu-\mathrm{H})_{2} \mathrm{~W}_{3}(\mathrm{CO})_{12}\left(\mathrm{PMe}_{3}\right)-\right.$ (NO)] (5). The ORTEP drawing of the anionic unit in 5 (5-) is shown in Figure 1. The two crystallographically independent molecules of $\mathbf{5}$ in the unit cell are only slightly different from each other, and only one of them is discussed here. The three tungsten atoms in $\mathbf{5}^{-}$reside in approximately octahedral environments. The two hydrides observed in the ${ }^{1} \mathrm{H}$ NMR spectra (Table 4) should bridge the $\mathrm{W}(1 \mathrm{~A}) / \mathrm{W}(2 \mathrm{~A})$ pair and $\mathrm{W}(1 \mathrm{~A}) / \mathrm{W}(3 \mathrm{~A})$ pair, respectively, since the $W(1 \mathrm{~A})-\mathrm{W}(2 \mathrm{~A})(3.492(2) \AA)$ and $\mathrm{W}(1 \mathrm{~A})-\mathrm{W}(3 \mathrm{~A})(3.422(2) \AA)$ distances fall within the range of a $3 \mathrm{c}-2 \mathrm{e}$ W-H-W bond, $3,4,16$ and the W(2A)-W(3A) $(5.344(2)$ $\AA$ ) distance indicates the absence of any metal-metal interaction. The spectra (vide infra) are also consistent with this assignment. The $\mathrm{NO}^{17}$ ligand, which is trans to one of the bridging hydrides and cis to the other, is linearly coordinated to the central tungsten atom. The $\mathrm{PMe}_{3}$ ligand is cis to the $\mathrm{NO}$ and both hydride ligands.

The X-ray crystal structure of $\left[\mathrm{HP}^{\prime} \mathrm{Bu}_{3}\right]\left[(\mu-\mathrm{H})_{2} \mathrm{~W}_{3}(\mathrm{CO})_{12^{-}}\right.$ $\left.\left(\mathrm{PMe}_{3}\right)(\mathrm{NO})\right]$ (5) serves as a complement to elucidate the structure of 3 as $\left[\mathrm{HPtBu}_{3}\right]\left[\left\{(\mu-\mathrm{H}) \mathrm{W}(\mathrm{CO})_{5}\right\}_{2}\left\{\mathrm{~W}(\mathrm{CO})_{3}(\mathrm{NO})\right\}\right]$ even though the poor quality of $3^{18}$ renders differentiation between $\mathrm{NO}$ and $\mathrm{CO}$ ligands questionable. The structures of $\mathrm{H}_{2} \mathrm{~W}_{3}$ $(\mathrm{CO})_{13}(\mathrm{NO})^{-}\left(^{-}\right)$and $\mathrm{H}_{2} \mathrm{~W}_{3}(\mathrm{CO})_{12}\left(\mathrm{PMe}_{3}\right)(\mathrm{NO})^{-}$(5-) $^{-}$are most similar to that of $\mathrm{HM}_{2} \mathrm{M}^{\prime}(\mathrm{CO})_{14}\left(\mathrm{M}=\mathrm{Re}, \mathrm{M}^{\prime}=\mathrm{Mn} ;{ }^{192} \mathrm{M}, \mathrm{M}^{\prime}\right.$ $=R e ;{ }^{19 b} M=M n, M^{\prime}=R^{19 c}$ ), which also has three metal atoms residing in a roughly octahedral environment and is also an open cluster with a bent skeleton. The latter contains a metal-metal bond and only one $3 \mathrm{c}-2 \mathrm{e}$ bond. The complex $\operatorname{Re}_{3}(\mu-\mathrm{H})_{2}(\mathrm{CO})_{10^{-}}$ $(\mathrm{L})_{2}{ }^{-}\left(\mathrm{L}=\mathrm{PPh}_{3},{ }^{20 \mathrm{a}} \mathrm{CO}^{20 \mathrm{~b}}\right)$, on the other hand, has two $3 \mathrm{c}-2 \mathrm{e}$ bonds and one metal-metal bond. It is interesting to note that the cluster skeleton of $\mathrm{W}_{3}(\mathrm{CO})_{14}{ }^{2-}$ was recently reported to be a chain of three tungsten atoms with octahedral coordination. ${ }^{21}$

The spectroscopic data (Table 4) for 3 are fully consistent with its formulation. Though $\mathrm{X}$-ray analysis could not differentiate $\left[\mathrm{HP}^{t} \mathrm{Bu}_{3}\right]^{+}$from $\mathrm{P}^{t} \mathrm{Bu}_{3}$, a characteristic one-bond coupling between $\mathrm{P}$ and $\mathrm{H}(J=444 \mathrm{~Hz})$ in both ${ }^{1} \mathrm{H}$ and ${ }^{31} \mathrm{P}$ NMR provides solid

(13) (a) Nato, C. C.; Kim, S.; Meng, Q.; Sweigart, D. A.; Chung, Y. K. J. Am. Chem. Soc. 1993, 115, 2077. (b) vander Graaf, T.; Hofstra, R. M. J.; Schlder, P. G. M.; Rijkhoff, M.; Stufkens, D. J.; vander Linden, J. G. M. Organometallics 1991, 10, 3668 . (c) Menon, R. K.; Brown, T. L. Inorg. Chem. 1989, 28, 1370. (d) Rigo, P.; Bressan, M.; Morvillo, A. J. Organomet. Chem. 1976, 105, 263.

(14) Monitoring of the reaction of $\mathrm{HW}_{2}(\mathrm{CO})_{7}(\mathrm{THF})_{2}(\mathrm{NO})$ with $\mathrm{Cp}_{2} \mathrm{Co}$ in THF- $d_{8}$ indicated the instantaneous formation of $\mathrm{HW}_{2}(\mathrm{CO})_{10^{-}}$and $\mathrm{H}_{2} \mathrm{~W}_{3}(\mathrm{CO})_{13}(\mathrm{NO})^{-}$

(15) Darensbourg, M. Y.; Slater, S. J. Am. Chem. Soc. 1981, $103,5914$. (16) Darensbourg, M. Y.; Ash, C. E. Adv. Organomet. Chem. 1987, $27,1$.

(17) The location of the NO ligand was distinguished from that of the $C O$ ligand by conspicuous discrepancies in the thermal parameters for the $\mathrm{N}$ and $\mathrm{C}$ atoms when incorrectly assigned. For instance, the reverse of $\mathrm{N}$ and $\mathrm{C}(1)$ gave the following thermal parameters $\left(B_{\text {iso, }}, \AA^{2}\right): \mathrm{N}(1 \mathrm{~A})$ $7.8(12) ; \mathrm{C}(1 \mathrm{~A}), 3.1(8) ; \mathrm{N}(1 \mathrm{~B}), 5.9(10) ; \mathrm{C}(1 \mathrm{~B}), 4.0(9)$. The reverse of $\mathrm{N}$ and $C(2)$ gave the following thermal parameters $\left(\mathrm{A}^{2}\right): \mathrm{N}(1 \mathrm{~A}), 8.3-$ (13); C(2A), 3.1(8); N(1B), 9.3(14); C(2B), 3.9(9). Hodgson, D. J.; Ibers, J. A. Inorg. Chem. 1968, 7, 2345.

(18) Lin, J. T. Unpublished research. Crystals were grown by cooling a solution of 3 in $\mathrm{CH}_{2} \mathrm{Cl}_{2} /$ hexane (1:5) at $-5^{\circ} \mathrm{C}$. X-ray crystal data for 3: $\mathrm{C}_{72} \mathrm{H}_{60} \mathrm{~N}_{6} \mathrm{O}_{42} \mathrm{P}_{3} \mathrm{~W}_{9}$, fw $=3356.37$; triclinic, $P 1 ; a=15.480(4), b=$ $17.645(3), c=21.889(8) \AA ; \alpha=73.36(2), \beta=100.68(3), \gamma=109.23$ (2) $; V=5382(3) \AA^{3} ; Z=2 ; 2 \theta_{\max }=45^{\circ}, R=0.069, R_{w}=0.066$ for $I>2 \sigma(I)$. The cation $\mathrm{HP}^{\prime} \mathrm{Bu}_{3}+$ was found to be disordered with a $65 \%$ occupancy.

(19) (a) Kaesz, H. D.; Bau, R.; Churchill, M. R. J. Am. Chem. Soc, 1967 89, 2775, (b) Yang, C. S.; Cheng, C. P.; Guo, L. W.; Wang, Y. J. Chin. Chem. Soc. 1985, 32, 17. (c) Bullock, R. M.; Brammer, L.; Schultz, A. J.; Albinati, A.; Koetzle, T. F. J. Am. Chem. Soc. 1992, 114, 5125 .

(20) (a) Beringhelli, T.; Ciani, G.; D'Alfonso, G.; Freni, M. J. Organomet. Chem. 1986, 311, C51. (b) Beringhelli, T.; Ciani, G.; D'Alfonso, G.; Molinari, H.; Sironi, A. Inorg. Chem. 1985, 24, 2666.

(21) Beletskaya, I.; Vaskoboynikov, A. Z.; Chuklanova, E. B.; Gusev, A. I.; Kisin, A. V. J. Organomet. Chem. 1993, 454, 1. 

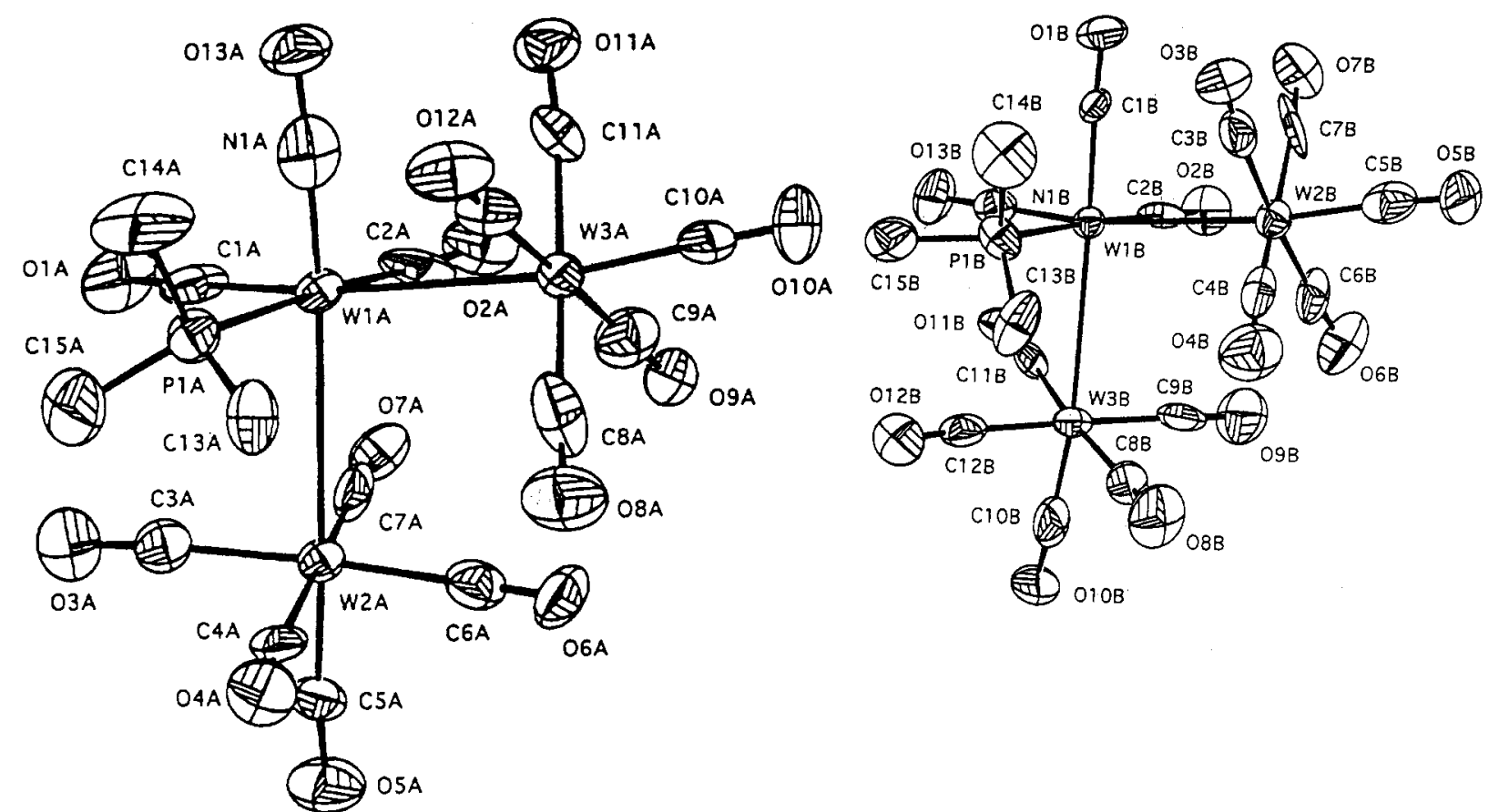

Figure 1. ORTEP drawing of molecules $A$ and $B$ in the anion of $\left[\mathrm{HP}^{t} \mathrm{Bu}_{3}\right]\left[(\mu-\mathrm{H})_{2} \mathrm{~W}_{3}(\mathrm{CO})_{12}\left(\mathrm{PMe}_{3}\right)(\mathrm{NO})\right](5)$. Thermal ellipsoids are drawn with $50 \%$ probability boundaries.

Table 4. ${ }^{1} \mathrm{H},{ }^{31} \mathrm{P}\{\mathrm{H}\}$, and ${ }^{15} \mathrm{~N}$ NMR Data

\begin{tabular}{|c|c|c|c|}
\hline compd & $\delta, a, b$ ppm $(J, \mathrm{~Hz})$ & $\delta,^{c} \operatorname{ppm}(J, \mathrm{~Hz})$ & $\delta, d \mathrm{ppm}(J, \mathrm{~Hz})$ \\
\hline 1 & $\begin{array}{l}1.64\left(\mathrm{~d}, 9 \mathrm{H},{ }^{3} J_{\mathrm{P}-\mathrm{H}}=12.0,{ }^{\mathrm{t}} \mathrm{Bu}\right),-11.4\left(\mathrm{~d}, 1 \mathrm{H},{ }^{2} J_{\mathrm{P}-\mathrm{H}}=8.7\right. \\
\left.1 J_{\mathrm{W}-\mathrm{H}}=51.2,33.4, \mu-\mathrm{H}\right)\end{array}$ & $87.0\left(\mathrm{~s},{ }^{1} J_{\mathrm{W}-\mathrm{P}}=240\right)$ & \\
\hline $3^{e}$ & $\begin{array}{c}5.89\left(\mathrm{~d}, 1 \mathrm{H},{ }^{1} J_{\mathrm{P}-\mathrm{H}}=444, H \mathrm{P}\right), 1.75\left(\mathrm{~d}, 9 \mathrm{H},{ }^{3} J_{\mathrm{P}-\mathrm{H}}=7.7,{ }^{\mathrm{t}} \mathrm{Bu}\right) \\
-9.88\left(\mathrm{dd}, 1 \mathrm{H},{ }^{2} J_{\mathrm{H}-\mathrm{H}}=4.5,{ }^{2} J_{\mathrm{N}-\mathrm{H}}=1.16,{ }^{1} J_{\mathrm{W}-\mathrm{H}}=48.5,38.9,\right. \\
\mu-\mathrm{H}),-13.0\left(\mathrm{dd}, 1 \mathrm{H},{ }^{2} J_{\mathrm{N}-\mathrm{H}}=2.36,{ }^{1} J_{\mathrm{W}-\mathrm{H}}=37.7,27.3, \mu-\mathrm{H}\right)\end{array}$ & $62.2(\mathrm{~s}, \mathrm{HP})$ & $\begin{array}{l}-8.86\left(\mathrm{dd},{ }^{2} J_{\mathrm{N}-\mathrm{H}}=2.36\right. \\
\left.\quad 1.16,1 J_{\mathrm{W}-\mathrm{N}}=97.7, N \mathrm{O}\right)\end{array}$ \\
\hline 4 & $\begin{array}{c}7.75-7.53(\mathrm{~m}, 30 \mathrm{H}, \mathrm{Ph}),-9.82\left(\mathrm{~d}, 1 \mathrm{H},{ }^{2} J_{\mathrm{H}-\mathrm{H}}=4.5,{ }^{1} J_{\mathrm{W}-\mathrm{H}}=48.5\right. \\
38.9, \mu-\mathrm{H}), 13.0\left(\mathrm{~d}, 1 \mathrm{H},{ }^{1} J_{\mathrm{H}-\mathrm{H}}=37.7,27.3, \mu-\mathrm{H}\right)\end{array}$ & 26.7 (s, PNP) & \\
\hline $5^{e}$ & $\begin{array}{c}5.92\left(\mathrm{~d}, 1 \mathrm{H},{ }^{1} J_{\mathrm{P}-\mathrm{H}}=447, H \mathrm{P}\right), 1.77\left(\mathrm{~d}, 9 \mathrm{H},{ }^{2} J_{\mathrm{P}-\mathrm{H}}=15.0, \mathrm{P} M e_{3}\right), \\
1.76\left(\mathrm{~d}, 9 \mathrm{H},{ }^{3} J_{\mathrm{P}-\mathrm{H}}=15.0,{ }^{\mathrm{t}} \mathrm{Bu}\right),-8.99\left(\mathrm{ddd}, 1 \mathrm{H},{ }^{2} J_{\mathrm{P}-\mathrm{H}}=17.5 \text {, }\right. \\
\left.1 J_{\mathrm{H}-\mathrm{H}}=4.00,{ }^{2} J_{\mathrm{N}-\mathrm{H}}=1.08,{ }^{1} J_{\mathrm{W}-\mathrm{H}}=52.8,45.3, \mu-\mathrm{H}\right),-12.4(\mathrm{ddd},\end{array}$ & $\begin{array}{r}62.1(\mathrm{~s}, 1 \mathrm{P}, \mathrm{HP}),-32.1(\mathrm{~s} \\
\left.\quad 1 \mathrm{P}, \mathrm{I}_{\mathrm{W}-\mathrm{P}}=223, P \mathrm{Me}_{3}\right)\end{array}$ & $\begin{aligned}-12.1\left(\mathrm{ddd},{ }^{2} J_{\mathrm{P}-\mathrm{N}}=5.04\right. \\
2 J_{\mathrm{N}-\mathrm{H}}=2.47,1.06 \\
\left.1 J_{\mathrm{W}-\mathrm{N}}=100, N O\right)\end{aligned}$ \\
\hline $6^{e}$ & $\begin{array}{c}7.63-6.62(\mathrm{~m}, 15 \mathrm{H}, \mathrm{Ph}), 5.86\left(\mathrm{~d}, 1 \mathrm{H}, 1 J_{\mathrm{P}-\mathrm{H}}=456, H \mathrm{P}\right), 1.76(\mathrm{~d}, \\
\left.9 \mathrm{H},{ }^{3} J_{\mathrm{P}-\mathrm{H}}=15.9,{ }^{\mathrm{t}} \mathrm{Bu}\right),-7.97\left(\mathrm{ddd}, 1 \mathrm{H},{ }^{2} J_{\mathrm{P}-\mathrm{H}}=16.3,{ }^{2} J_{\mathrm{H}-\mathrm{H}}=3.6,\right. \\
\left.2 J_{\mathrm{N}-\mathrm{H}}=1.02,1 J_{\mathrm{W}-\mathrm{H}}=54.1,47.6, \mu-\mathrm{H}\right),-11.3\left(\mathrm{ddd}, 1 \mathrm{H},{ }^{2} J_{\mathrm{P}-\mathrm{H}}=\right. \\
\left.14.8,{ }^{2} J_{\mathrm{N}-\mathrm{H}}=2.70,1 J_{\mathrm{W}-\mathrm{H}}=49.8,42.2, \mu-\mathrm{H}\right)\end{array}$ & $\begin{array}{l}62.2(\mathrm{~s}, 1 \mathrm{P}, \mathrm{HP}), 17.0(\mathrm{~s} \\
\left.\quad 1 \mathrm{P},{ }^{1} J_{\mathrm{W}-\mathrm{P}}=379, \mathrm{PPh}_{3}\right)\end{array}$ & $\begin{array}{l}-12.2\left(\mathrm{ddd},{ }^{2} J_{\mathrm{P}-\mathrm{N}}=4.52\right. \\
2 J_{\mathrm{N}-\mathrm{H}}=2.73,1.08 \\
\left.{ }^{1} J_{\mathrm{W}-\mathrm{N}}=99.9, N O\right)\end{array}$ \\
\hline 7 & $\begin{array}{l}5.85\left(\mathrm{~d}, 1 \mathrm{H},{ }^{1} J_{\mathrm{P}-\mathrm{H}}=444, \mu-\mathrm{H}\right), 4.16\left(\mathrm{~m}, 6 \mathrm{H}, \mathrm{CH}_{2}\right), 1.82(\mathrm{~d}, 9 \mathrm{H}, \\
\left.3 J_{\mathrm{P}-\mathrm{H}}=15.0,{ }^{\mathrm{t} B u}\right), 1.33\left(\mathrm{t}, 9 \mathrm{H},{ }^{3} J_{\mathrm{H}-\mathrm{H}}=12.0, \mathrm{CH}_{2} \mathrm{CH}_{3}\right),-9.13(\mathrm{dd}, \\
\left.1 \mathrm{H},{ }^{2} J_{\mathrm{P}-\mathrm{H}}=21.3,{ }^{2} J_{\mathrm{H}-\mathrm{H}}=4.38,{ }^{1} J_{\mathrm{W}-\mathrm{H}}=53.1,45.3, \mu-\mathrm{H}\right),-12.6(\mathrm{dd}, \\
\left.1 \mathrm{H},{ }^{2} J_{\mathrm{P}-\mathrm{H}}=21.4,{ }^{1} J_{\mathrm{W}-\mathrm{H}}=47.2,32.9, \mu-\mathrm{H}\right)\end{array}$ & $\begin{array}{l}62.1(\mathrm{~s}, 1 \mathrm{P}, \mathrm{HP}), 130(\mathrm{~s}, \\
\left.\quad 1 \mathrm{P}, 1_{\mathrm{W}-\mathrm{P}}=365, P(\mathrm{OEt})_{3}\right)\end{array}$ & \\
\hline 8 & $\begin{array}{l}5.90\left(\mathrm{~d}, 1 \mathrm{H},{ }^{1} J_{\mathrm{P}-\mathrm{H}}=447, H \mathrm{P}\right), 3.79(\mathrm{~s}, 3 \mathrm{H}, M e N C), 1.84(\mathrm{~d}, 9 \mathrm{H}, \\
\left.3_{J_{\mathrm{P}-\mathrm{H}}}=14.7,{ }^{\mathrm{B} u}\right),-9.60\left(\mathrm{~d}, 1 \mathrm{H},{ }^{2} J_{\mathrm{H}-\mathrm{H}}=4.75,1 J_{\mathrm{W}-\mathrm{H}}=53.6,44.4,\right. \\
\mu-\mathrm{H}),-12.9\left(\mathrm{~d}, 1 \mathrm{H}, 1{ }^{1} J_{\mathrm{W}-\mathrm{H}}=45.0,30.0, \mu-\mathrm{H}\right)\end{array}$ & $62.5(\mathrm{~s}, 1 \mathrm{P}, \mathrm{HP})$ & \\
\hline
\end{tabular}

${ }^{a}$ All are measured in acetone- $d_{6}{ }^{b} \delta$ values relative to $\mathrm{Me}_{4} \mathrm{Si}$. ${ }^{c} \delta$ values relative to $85 \% \mathrm{H}_{3} \mathrm{PO}_{4}(\mathrm{aq}) .{ }^{d} \delta$ values relative to neat $\mathrm{MeNO}$ : low-field positive, measured at $25^{\circ} \mathrm{C}$. ${ }^{e}$ The sample is ${ }^{15} \mathrm{~N}$ enriched, and all enriched samples were prepared from $\mathrm{HW}_{2}(\mathrm{CO})_{9}\left({ }^{15} \mathrm{NO}\right)$. Abbreviations: ax $=$ axial, eq $=$ equatorial.

evidence for the ionic nature of 3 . The two hydrides are mutually cis $\left({ }^{2} J_{\mathrm{H}-\mathrm{H}}=4.5 \mathrm{~Hz}\right)$, and both have two sets of tungsten satellites $\left(J_{\mathrm{W}-\mathrm{H}}=38.9,48.5 \mathrm{~Hz}\right.$ and $\left.27.3,37.7 \mathrm{~Hz}\right)$ in the ${ }^{1} \mathrm{H}$ NMR spectra. The presence of a NO ligand is evident on the basis of a NO stretching at $1652 \mathrm{~cm}^{-1}$ in the infrared and one chemical shift at $\delta_{\mathrm{N}}-8.86 \mathrm{ppm}\left(J_{\mathrm{N}-\mathrm{W}}=97.7 \mathrm{~Hz}\right)$ in the ${ }^{15} \mathrm{~N} \mathrm{NMR}$ for ${ }^{15} \mathrm{~N}$-enriched 3. This NO ligand should be coordinated to the central tungsten atom W(2) since it is coupled to both hydrides. According to our previous studies on a series of dimeric W-H-W complexes containing a NO ligand, ${ }^{4}$ we assign the two hydrides in 3 as being cis $\left(\delta_{\mathrm{H}}=-9.83 \mathrm{ppm}, J_{\mathrm{H}^{1}{ }^{15 \mathrm{~N}}}=1.16 \mathrm{~Hz}\right)$ and trans $\left(\delta_{\mathrm{H}}=-13.0\right.$ ppm, $J_{\mathrm{H}_{-}{ }^{15} \mathrm{~N}}=2.36 \mathrm{~Hz}$ ) to the NO ligand, respectively, on the basis of several observations: (1) the chemical shift of the cis hydride $(\delta=-9.83 \mathrm{ppm})$ appears at lower field than that of the trans hydride $(\delta=-13.0 \mathrm{ppm})$ by about $3 \mathrm{ppm}$; (2) the $\mathrm{N}-\mathrm{H}$ coupling constant of the cis hydride is ca. $1 \mathrm{~Hz}$ and that of the trans hydride is ca. $2 \mathrm{~Hz}$; (3) the $\delta_{\mathrm{N}}$ value $(-8.9 \mathrm{ppm})$ of 3 appears at lower field than those for complexes which have NO trans to a $\mu-\mathrm{H}(\delta=-16.0$ to $-24.0 \mathrm{ppm})$ and at higher field than those for complexes which have NO cis to a $\mu-\mathrm{H}(\delta=5.5$ to $-3.0 \mathrm{ppm})$.

Chemical Reactivity. Complex 3 was found to be reactive toward nucleophiles such as phosphines and isocyanides (eq 3 ).

$$
\begin{gathered}
(\mu-\mathrm{H})_{2} \mathrm{~W}_{3}(\mathrm{CO})_{13}(\mathrm{NO})^{-}+\mathrm{L} \underset{(\mu-\mathrm{H})_{2} \mathrm{~W}_{3}(\mathrm{CO})_{12}(\mathrm{~L})(\mathrm{NO})^{-}}{\stackrel{\mathrm{CH}_{2} \mathrm{Cl}_{2}}{\rightarrow}} \\
\mathrm{L}=\mathrm{PMe}_{3}, \mathrm{PPh}_{3}, \mathrm{P}(\mathrm{OEt})_{3}, \mathrm{MeNC}
\end{gathered}
$$

Complexes 5-8 were isolated and were found to have the formulation $\left[\mathrm{W}(\mathrm{CO})_{2}(\mathrm{NO})(\mathrm{L})\right]\left[(\mu-\mathrm{H}) \mathrm{W}(\mathrm{CO})_{5}\right]_{2}$ (vide supra). A large excess of MeNC should be avoided for the synthesis of 8 since it was found that 8 reacted further with MeNC to form 
$\mathrm{HW}_{2}(\mathrm{CO})_{7}(\mathrm{MeNC})_{2}\left(\mathrm{NO}_{\mathrm{eq}}\right) .^{22}$ It is evident that the NO ligand plays an important role in activating the coordinated $\mathrm{CO}$ ligand. Our previous studies on dinuclear $\mathbf{M}-\mathrm{H}-\mathbf{M}$ complexes unanimously have ligand substitution occur at the metal attached to the NO ligand.,4,5,7,23

The spectroscopic data for complexes $5-8$ are also consistent with their formulation. The hydride which is cis to the NO ligand appears at lower field ( $\delta=-8.0$ to $-10.0 \mathrm{ppm})$ than that which is trans to the NO ligand $(\delta=-11.3$ to -13.0$)$ by ca. $3 \mathrm{ppm}$ in the ${ }^{1} \mathrm{H}$ NMR spectra. Both hydride ligands are cis to the phosphine ligand for 5-7, and the two-bond phosphorus-hydrogen coupling constants range from 15 to $21 \mathrm{~Hz} .{ }^{24}$ Each hydride has two sets of tungsten satellites, and the coupling constant, $1 J_{\mathrm{H}-\mathrm{W}}$, ranges from 30 to $54 \mathrm{~Hz}$. ${ }^{15} \mathrm{~N}$-enriched 5 and 6 have ${ }^{2} J_{\mathrm{N}-\mathrm{H}}$ (cis) at $1.0 \mathrm{~Hz}$ and ${ }^{2} J_{\mathrm{N}-\mathrm{H}}$ (trans) at $2.5 \mathrm{~Hz}$, respectively. The chemical shift of NO also appears in a reasonable range $(\delta=-12.1$ for 5 and -12.2 for 6 ) in the ${ }^{15}$ N NMR spectra. The one-bond tungstenphosphorus coupling constants, ranging from 220 to $380 \mathrm{~Hz}$, are consistent with those frequently observed. 25

Our preliminary results indicated that $\mathrm{HW}(\mathrm{CO})_{5}$-reacted with $\mathrm{HW}_{2}(\mathrm{CO})_{8}\left({ }^{\mathrm{PrNC}}\right)(\mathrm{NO})^{19}$ and $\mathrm{HMO}_{2}(\mathrm{CO})_{9}(\mathrm{NO})$ to form

(22) Lin, J. T.; Chen, C. C.; Huang, P. S.; Hong, F. E.; Wen, Y. S. Organometallics $1993,12,406$.

(23) Lin, J. T.; Cheng, K. Y.; Gong, M. L.; Chang, C. C.; Gau, H. M.; Tsen, L. H.; Wen, Y.S. J. Organomet. Chem. 1992, 431,65.

(24) Darensbourg, M. Y.; El Mehdawi, R.; Delord, T. J.; Fronezek, F. R. Watkins, S. F. J. Am. Chem. Soc. 1984, 106, 2583

(25) Pregosin, P. S.; Kunz, R. W. ${ }^{1} P$ and ${ }^{13} C$ NMR of Transition Metal Phosphine Complexes; Springer-Verlag: New York, 1979.
$\left[\mathrm{W}(\mathrm{CO})_{2}(\mathrm{PrNC})(\mathrm{NO})\right]\left[(\mu-\mathrm{H}) \mathrm{W}(\mathrm{CO})_{5}\right]^{-}$and $(\mu-\mathrm{H})_{2} \mathrm{Mo}_{2} \mathrm{~W}-$ $(\mathrm{CO})_{13}(\mathrm{NO})^{-}$, respectively. The reaction of $\mathrm{HFe}(\mathrm{CO})_{4}{ }^{-}$with $\mathrm{HW}_{2}(\mathrm{CO})_{9}(\mathrm{NO})$, on the other hand, led to the formation of $\mathrm{HFeW}(\mathrm{CO})_{9}{ }^{-26}$ and $\mathrm{Fe}(\mathrm{CO})_{3}(\mathrm{NO})^{-}$. The mechanistic aspect of the formation of 3- as well as the synthesis of related hydrogenbridged heterobimetallic complexes ${ }^{27}$ is currently under investigation.

Acknowledgment. We thank the National Science Foundation of the Republic of China for financial support (Grants NSC 82-0208-M-001-039 and NSC 83-0115-C-001-0016) and Ms. Su-Ching Lin and Ms. Mei-Man Chen for excellent NMR technical assistance.

Supplementary Material Available: Pluto drawings for $\left[(\mu-\mathrm{H})_{2} \mathrm{~W}_{3}\right.$ $\left.(\mathrm{CO})_{13}(\mathrm{NO})\right]^{-}\left(3^{-}\right)$and the cations, $\left[\mathrm{HP}^{+} \mathrm{Bu}_{3}\right]^{+}$, of 5 , listings of crystallographic data, all bond distances and angles, anisotropic thermal parameters and isotropic thermal parameters, and positional parameters for calculated hydrogen atoms for 5 , and listings of positional parameters and $B_{\text {iso }}$ values, anisotropic thermal parameters and isotropic thermal parameters, and bond distances for 5 when $\mathrm{N}(1)$ and $\mathrm{C}(1)$ are reversed and when $N(1)$ and $C(2)$ are reversed (27 pages). Ordering information is given on any current masthead page.

(26) Arndt, L.; Delord, T.; Darensbourg, M. Y. J. Am. Chem. Soc. 1984, $106,456$.

(27) Balbach, B.; Baral, S.; Biersack, H.; Hermann, W. A.; Labinger, J. A; Scheldt, W. R.; Timmers, F. J.; Ziegler, M. L. Organometallics 1988, 7,325 . 